ANALOGIE ET HOMOLOGIE DANS L'ACCENTUATION_DE L' ANGLAIS

par L. GUIERRE

Paris, Sorbonne, Mai 1967 


\section{ANALOGIE ET HOMOLOGIE DANS L'ACCENTUATION DE L'ANGLAIS}

\section{Terminaisons Faibles et Terminaisons Fortes}

Le système accentuel de 1'anglais (accent de mot) peut être décrit essentiellement, semble-t-il, à partir de l'opposition entre :

Terminaisons Faibles et Terminaisons Fortes

On appellera "forte" une terminaison qui impose un schéma accentuel constant (qu'il existe ou non une forme dérivante et quel que soit le sahéma accentuel de cette forme dérivante). Ainsi 〈-ic〉 est forte puisque 1'on a toujours /-20/ (accent principal sur 1'avant-dernière syllabe) : crǐtic, elěctric, enı̆gma $\rightarrow$ enigmătic, măgnet $\rightarrow$ magnětic .... (1).

On dira que les terminaisons fortes sont hanifestation d'une force appelée analogie. Cela signifie, par exemple que tous les mots en 〈-ion〉 présente et, dans certaines limites, à venir, seront accentués $/-20 /$ par analogie avec les quelques 2.000 mots attestés dans le corpus et accentués selon ce schéma.

On appelera "faible" une terminaison qui, ajoutée à un dérivant, n'en modifie pas l'accentuation.

Ainsi $\langle-e r\rangle,\langle-a b l e\rangle,\langle-1 y\rangle$ sont faibles puisque $1^{\prime}$ 'on a cǒmfort cơmforter, cơmfortable, cǒmfortably; jưstify jǔstifiable, jưstifiably $\ldots$...

On dira qu'un mot en 〈-ly〉 sera nécessairement accentué, par homologie, comme I'adjectif dérivant (adǒrably, fơrtunately, immědiately, concisely....).

A partir de cette belle et pure dichotomie, qui fait penser à un language artificiel, on pourrait espérer que l'analyste n'aura même pas à hésiter ni à choisir dans sa description; tous les mots viendront docilement

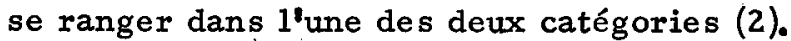

Or cette dichotomie, même si elle est valable dans un très grand nombre de cas, ne résoud pas d'emblée tous les problèmes. On trouvera

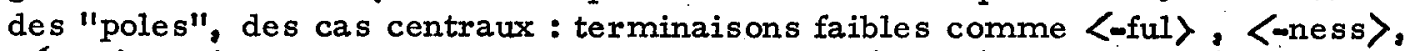
$\langle$-ing $\rangle,\langle-e d\rangle$, terminaisons fortes comme $\langle-i t y\rangle$, ou $\langle-i c a l\rangle$, mais on verra qu'il existe fort peu de classes intégralement analogiques ou intégralement homologiques. Le plus souvent une dominante statistique très nette se manifestera, souvent le partage sera très inégal. 
Exemple 1 : Les mots en 〈wical> forment une classe analogique. Ils sont accentués $/-200 /$. On trouve cependant au moins une exception (sur 600 mots environ) : le mot <umbilical> /1020/ [-'laik1] (variante) qui ne peut être rattaché qu'à son homologue, le nom 〈umbilicus $>/ 1020 /$ ['laikas] (variante).

Exemple 2 : La règle de "lion" : $\left\langle-\mathrm{iV}^{\mathrm{n}}\left(\mathrm{C}^{\mathrm{n}}\right)\right\rangle \Rightarrow /-2(0) 0 /$. Cette règle (3) signifie que 1'accent / $/$ (accent principal) précède immédiatement la terminaison graphique :

$\langle i+$ une ou plusieurs voyelles $t$, facultativement, une ou plusieurs consonnes >; $1^{2}$ élision d'une syllabe, symbolisée par (0) étant gouvernée par des règles comme celles de la palatalisation et celles de la réduction de $[i]$ à $[\underline{I}]$ ou $[\mathbf{j}]$.

* [laeksian $] \rightarrow\left[\right.$ exemples $:^{*}[$ [impia s $] \rightarrow[$ impjas $]:^{*}[$ ['glo:rias $] \rightarrow[$ 'glo:rias $]$

Cette règle analogique et son corollaire régissent en fait 4,000 mots environ. Les finales attestées sont au nombre de 40 environ, les plus

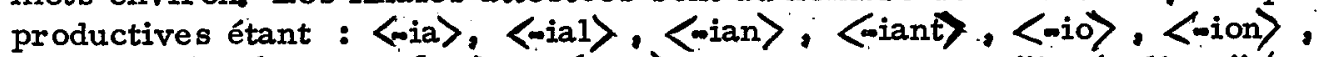

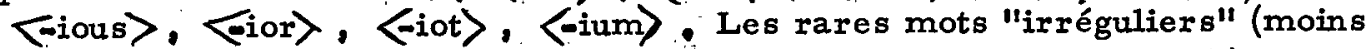
de $1 \%$ ) sont surtout, outre quelques emprunts étrangers non-assimilés (mafí, latakia, ratafia ....) des dérivés homologiques de verbes en $\langle-\mathrm{y}\rangle$ : esplal, decrial, defiant, reliant ....

Si <reliant) $s^{\prime} a c c e n t u e / 020 /$ [ri'laiant] c'est qu'il est rattaché à $\langle$ (to) rely $\rangle / 02 /\left[\mathrm{ri}^{1} \mathrm{lai}_{\mathrm{i}}\right]$. Si $\langle$ brilliant $\rangle$, par contre, s'accentue $/ 20 /$ ['briljont] et subit la pression analogique des mots en $\langle-i a\rangle,\langle-i a n\rangle, .66 !$ $c^{2}$ est qu'on ne peut le rattacher à aucun verbe.:

La règle de "lion" et son corollaire s'appliquent aussi au schéma

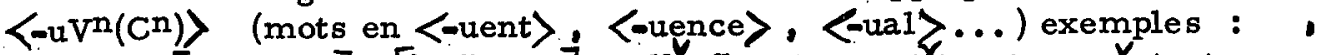
influence ['influans] $\rightarrow$ ['inflwans], mellufluent; constituent, accěntratm gargatuan, annual .... Les quelgues mots irréguliers sont, : des dérivés : subdüe $\rightarrow$ subdưlal; spirit $\rightarrow$ spiritual.

\subsection{Forme et Fonction}

Supposons une terminaison définie formellement par sa graphie (ainsi 〈-ive $\rangle$ ou $\langle-1 y\rangle$ ou $\langle-s o m e\rangle)$ ), il arrive souvent qu'à une seule forme graphique correspondent plusieurs phonies et/ou plusieurs fonctions syntaxiques ( $\mathrm{cf}$. l'exemple classique en français : les poulesidu couvent couvent)

Dans notre corpus on troure : 


\begin{tabular}{|c|c|c|}
\hline $\begin{array}{l}\text { attractive (adj.) } \\
\text { supply (adv.) } \\
\text { troubles ome (adj.) }\end{array}$ & $\begin{array}{l}{[-i v]} \\
{[-1 i]} \\
{[-s a m]}\end{array}$ & $\begin{array}{l}\text { arrive (v.) } \\
\text { supply (v.) } \\
\text { chromosome (n.) }\end{array}$ \\
\hline
\end{tabular}

On trouvera, de mème, que $\langle-i s h\rangle$ terminaison adjectivante est faible (classe homologique) alors que $\langle-i s h\rangle$ terminais on verbale est forte et produit une classe analogique.

Il peut donc être indispensable, pour définir complètement une terminaison, d'en donner la graphie, la phonie et la fonction si $1^{\prime}$ on veut éviter d'introduire certaines ambiguités dans la description de l'accent de mot. La recherche automatique a tenu compte de ces interférences entre les trois systèmes $(10)$.

\section{2:: Décomposition en classes}

Ici encore la synchronie se distingue nettement de la diachronie. Les terminaisons $\langle$-ious $\rangle,\langle-i o n\rangle,\langle-i t y\rangle$; forment synchroniquement des classes homogènes (4). Cependant certains auteurs, influencés sans doute par des considérations historiques, proposent de décrire chacune d'entre

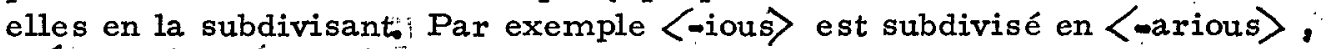

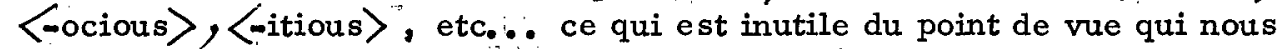
occupe. Par contre, quand une classe comme <-ive> ne semble pas régie par un principe dominant (analogie ou homologie) on tentera, à juste titre, de la décomposer. On ne pourra analyser <-ive> synchroniquement qu'en distinguant $\langle$-ative $\rangle,\langle$-itive $\rangle,\langle$-utive $\rangle$, etc., 6 (voir plus bas parag. 14,11$)$.

\subsection{Conflits}

Des conflits de nature diverse peuvent surgir :

a) Au sein même des modèles de dérivation, dans le cas où un dérivé peut être, à première vue, rattaché à deux dérivants d'accentuation différentes.

exemple : D. Jones donne pour 〈certification> soit $/ 10020 /$ soit /01020/ (c'est le seul mot de ce type présentant cette opposition), mais il précise :

/10020/, act of certifying; /01020/, providing with a certificate. (variante): :

Nous dirons : certify $/ 200 / \rightarrow$ certification $/ 10020 /$ certificate $/ 0200 / \rightarrow$ certification $/ 01020 /$ 
$L^{\prime}$ existence de variantes sera donc parfois (bien que $1^{\text {toccasion }}$ $s^{\prime}$ en présente rarement) justifiée par des considérations lexico-sémantiques.i Cependant la notion de "related form" (5), de "proximité du dérivant" ou de "dérivant le plus proche" demande, on le voit, à être précisée.

b) quand une chaine de dérivations forme une "boucle".

Ainsi telephony fait partie de la classe analogique 〈-phony > /-200/. Si 1 'on choisit 〈-phony> comme point de départ on obtient par dérivation (6)

\begin{tabular}{|c|c|c|c|c|}
\hline $\begin{array}{c}\langle- \text { phony }\rangle \\
\mid-200 /\end{array}$ & $\begin{array}{l}\text { (-phonist } \\
\text { (-phonize } \\
(- \text { phonous }\end{array}$ & $\begin{array}{l}\mid-200 / \\
/-200 / \\
/-200 /\end{array}$ & $\begin{array}{l}\text { de même } \\
\text { que }\end{array}$ & 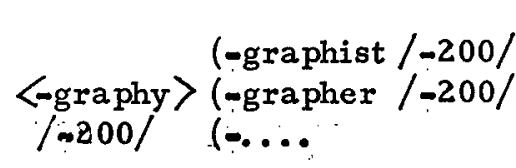 \\
\hline
\end{tabular}

ou encore

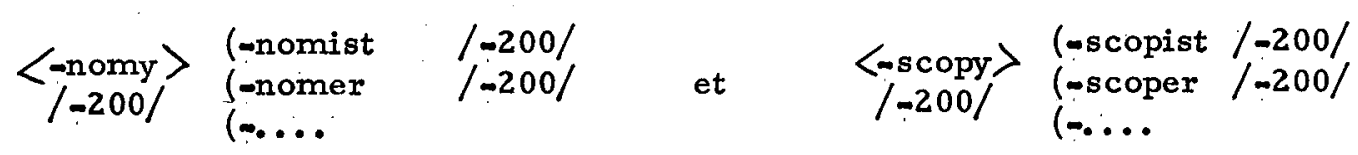

Par contre, de nombreux éléments monosyllabiques tels que

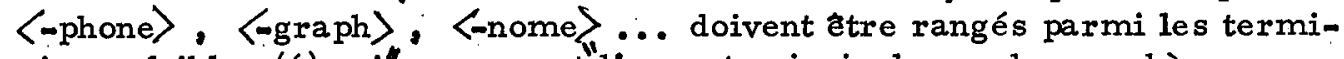

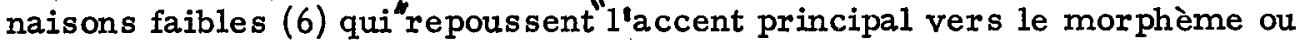
quasi-morphème initial.

exemples : tělephone, electrograph, dy̌namograph, mětronome...

Dans le cas, heureusement ou plutôt nécessairement exceptionnel,

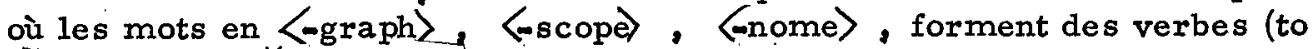
tělephone, to têlegraph) , on entrevoit immédiatement la latitude qu'aura la langue de créer, par dérivation, des termes en $\langle$-er $\rangle$.

Mais alors aura-t-on telepphony $\rightarrow$ ? telephoner $/ 0200 /$ ou tèlephone $\rightarrow$ ? tělephoner $/ 2000 /$

ou encore telègraphy $\rightarrow$ ? telègrapher /0200/

ou tèlegraph $\rightarrow$ ? tělegrapher $/ 2000 /$

D. Jones répond : tělephoner et telěgrapher ! ! !

On peut schématiser cette situation ainsi :

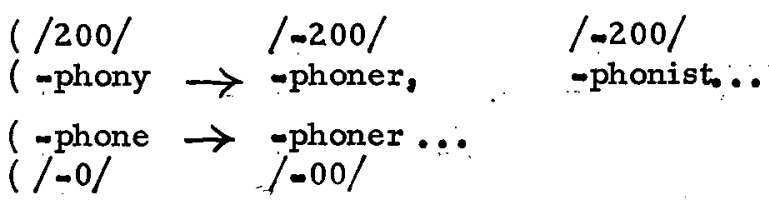


Le conflit est latent (7) dans la mesure où deux mécanismes généraux de la langue produisent des effets contradictoires. Cette situation ne peut que freiner le développement de certains schémas de dérivation.

En fait, les verbes du type 〈telephone〉 sont peu nombreux et certains sont peu usités. Les dérivés en 〈-er〉 entrent d'ailleurs en concurrence avec les dérivés en 〈-ist) (c. a, d. ici, en 〈-phonist〉, $\langle-$ graphist $\rangle$, 〈-nomist> ...) qui, eux, sont àccentuellement déterminés. L'analyste sera donc amené à postuler que certaines dérivations doivent être orientées ou ordonnées, et à constater que d'autres sont préférentielles et d'autres encore condamnées d'avance comme non-viables.

c) quand la règle de dérivation aboutit à des structures accentuelles inacceptables.

Ainsi on a trouvé (8) que la séquence accentuelle /-12// $n^{2}$ était pratiquement jamais attestée dans le système de $D$. Jones. Une des rarissimes exceptions (ou bien est-ce une faute dimpression ?) est la variante /012/ de exchangee (également accentué /102/). En qualité de dérivé du verbe 〈exchange〉 $/ 02 /$, on conçoit que 〈exchangee〉 conserve,

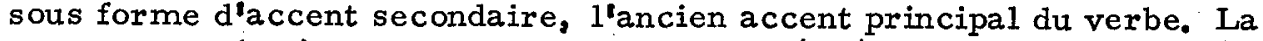
finale forte <-ee> produisant l'accentuation / $/ 2 /$, le résultat serait /012/ qui est, en principe absolument rejeté par le système. En fait le conflit devra être résolu c'est-à-dire qu'une règle devra l'emporter sur l'autre.

d) quand apparaft un danger de confusion formelle entre différentes terminaisons :

Ainsi pour la classe 〈-osis〉 1'analogie joue de façon rigoureuse (neurǒsis, psychǒsis, tubérculơsis ... ont tous $/-20 \Lambda \mathrm{Par}$ ailleurs la classe de 〈-morphosis〉, analogique elle aussi, produit / $200 /$. Cela semble indiquer que (de même que pour 〈-cracy〉 qui se sépare de l'ensemble $\{\langle-a c y\rangle\}$ et s'en distingue comme une sous-classe homogène), la classe 〈-morphosis〉 doit être analysée indépendamment de celle des "autres mots en 〈-osis〉".

D. Jones donne deux accentuations pour 〈metamorphosis〉:/10200/ et $/ 10020 /$. La situation de <-morphosis〉 n'est en effet pas très simple.: Des pressions latérales semblent s'exercer non seulement de la part des deux classes déjà mentionnées ( <-morphosis〉 et "autres mots en 〈-osisy').! mais aussi de la part des terminaisons <-morphy>, «-morphous〉, $\langle$-morphism $\rangle ..$. qui produisent toutes $/-20 \%$. La terminaison $\langle$-morphosis est donc placée à un véritable carrefour d'influences inconciliables qui

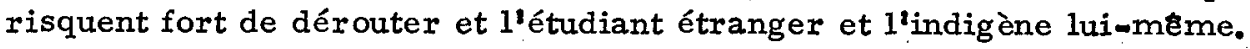


contradictoires.

e) quand deux classes analogiques imposent des accentuations

Comment se comportent dans ce cas les mots qui appartiennent à la fois aux deux classes?

On sait par exemple, que 1'accentuation des mots en 〈ic〉est $/-20 /$ : On sait aussi que $1^{\prime}$ accent précède immédiatement la finale $\left\langle-i V^{n}\left(C^{n}\right)\right\rangle$. Cette règle graphique, une fois transposée en termes phonétiques, reste valable, $\langle i>$ devenant $[i],[i]$ ou $[j]$. Comment réagissent les mots en $[-i i k]$ ?

On trouve deux mots de ce type : 〈ichthyic〉, [ijk $\left.\theta_{i i k]}\right]$ et 〈nucleic〉, ['nju:kliik]. Ces deux mots se séparent de leurs analogues en $\langle-i c\rangle$ pour rejoindre les mots de la classe analogique $\left\langle-i V^{n}\left(C^{n}\right)\right\rangle \cdot 1$ Nucleic et ichthyic ne peuvent évidemment pas satisfaire à la fois aux deux règles, La règle de "lion" domine la règle $\langle-$ ic $\rangle \Rightarrow /-20 /$.

On verra, et l'on pouvait préssentir, que tous ces conflits sont nécessairement limités: Sinon la langue en tant qu'instrument de commu-

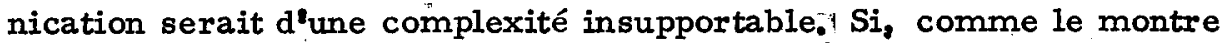
1 'expérience, ces conflits sont limités, c'est que la langue àccepte un certain nombre de contraintes ou de règles du type par exemple : "le dérivé 〈evǒcative〉 ne peut être rattaché à la fois à 〈evǒke〉 et à 〈 ěvocate〉; donc il ne devrait pas exister de couples de verbes du type 〈evơke〉 et < ̌rvocate> (c'est en fait le seul cas attesté), et l'existence de 〈evǒke〉 bloquera le développement du type 〈evocate〉(rare ?) et cela malgré 1'existence attestée de $\langle$ evocation $\rangle$.

Par contre, certains conflits une fois résolus, c'est-à-dire dès qu'on aura établi la hiérarchie des règles rien ne pourra objectivement freiner le développement de certaines classes : appoint $/ 02 / \rightarrow$ appointee/1 02/ et donc exchange $/ 02 / \rightarrow$ exchangee $/ 102 /$, une fois rejetée la variante /012/ qui est contraire aux règles des structures accentuelles (cf. parag.1 3.: $\left.2.11_{0}\right)$

\section{4. Absence de Dérivants}

Supposons que 1'analyste, en se fondant sur les faits et les statistłqueg, décide de décrire une classe comme homologique : la majorité des mots de cette classe étant obtenue par dérivation comme c'est le cas

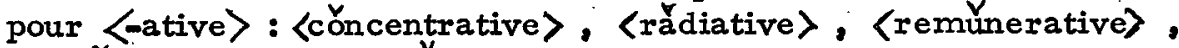

〈informative〉, 〈exclámative〉... L'expérience montre qu'il est rare que tous les dérivés possèdent un dérivant attesté (9): II semble que lorsque le nombre de dérivants attestés en langue contemporaine "décroit" dans 
une classe donnée, cette classe tende à "devenir" analogique et, vice-versa, quand le nombre de dérivants atte stés est élevé, la classe tende à "devenir" homologique. Il demeure que certains membres d'une classe nettement homologiques manquent de dérivants. I'analyste $n$ 'en est pas pour autant désarmé : d'autres procédés d'analyse s'offrent à lui. 1 Il utilisera par exemple, non pas un processus de dérivation simple êt linéaire (〈apology) $\rightarrow$ 〈apologize>) mais le procédé des "réseaux"! analogiques ou homologiques comportant au besoin des cases vides, c'est-à-dire, des formes "potentielles" $(10)$.

Exemple: les mots en $\langle-l o g y\rangle,\langle-$ graphy $\rangle,\langle-$ scopy $\rangle$,

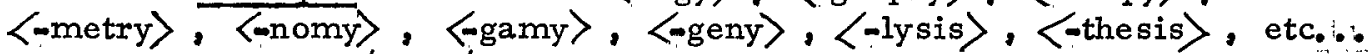
sont tous accentués $/-200 /$. Si on considère ces mots comme des formants déterminés analogiquement et, d'autre part, les terminaisons faibles : 〈-er〉, $\langle-$ ous $\rangle,\langle-$-al $\rangle,\langle-$ ent $\rangle,\langle-$ ence $\rangle,\langle-$ ize $\rangle,\left\langle-i s t t^{\prime}\right\rangle,\langle-$ ism $\rangle ..$ on peut établir un schéma générateur à deux dimensions qui permet de former des mots accentuellement déterminés en :

$$
\langle\text {-logist }\rangle,\langle\text { grapher }\rangle,\langle- \text { gamous }\rangle,\langle\text {-phonize }\rangle,\langle\text {-scopal }\rangle . .
$$

La langue contemporaine est loin d'utiliser pleinement toutes les possibilités de ce processus générateur, possibilités que nous considérerons comme faisant partie du stock disponible et accentuellement prëdéterminé. De plus des "cases vides" peuvent apparaftre en n'importe quel endroit de ce tableau y compris parmi les formants. On les remplira à l'aide de formes potentielles symbolisées $\left({ }^{\circ}\right)$ :

$$
\begin{aligned}
& \text { soliloquy } \rightarrow \text { soliloquist , 'soliloquent } \\
& \text { oeloquy } \rightarrow \text { eloquist }, \text { eloquent } \\
& \text { oantagony } \rightarrow \text { antagonist , antagonism }
\end{aligned}
$$

relation :

Le même type de procédé permettrait d'expliquer la double

discuss $/ 02 / \leftrightarrow \bullet$ discussive $/-20 / \leftrightarrow$ discussion $/-20 /$

-repercuss $/ 102 / \leftrightarrow$ repercussive $/-20 / \leftrightarrow$ repercussion $/-20 /$

\section{5. Neutralisation}

L'opposition entre analogie et homologie peut même se trouver neutralisée : certaines classes peuvent être décrites comme analogiques ou comme homologiques de façon tout aussi satisfaisante. C'est le cas de <-ical> qui peut être décrite soit comme une classe analogique $(/-200 /)$ soit comme une classe homologique de dérivés des membres de la classe analogique $\langle-i c\rangle$

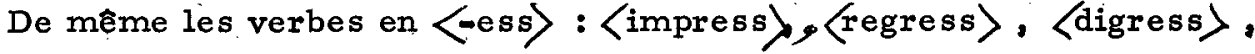


〈progress〉... /-2/ forment des adjectifs en 〈-essive〉/-20/. Tous les

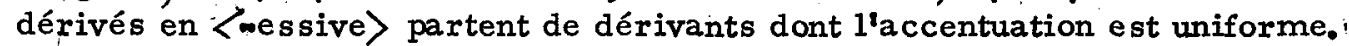
Dans ce cas le choix de l'analyse sera dicté par la possibilité de généralisér, d'étendre la règle au-delà de la sous-classe considérée.

\section{I. Les formants}

La réduction des dérivés appartenant à des classes homologiques doit normalement produire des éléments de base que $1^{\prime}$ on appelera mor phèmes, quasi-mor phèmes, pseüdo-morphèmes (II), (on néglige provisoirement la préfixation). Tous ces éléments seront appelés des "formants". L'analyste peut espérer que les formants seront régis :

a) par des règles analogiques qui sont des règles phonématiques séquentielles,

b) par des règles de structure accentuelle.

Ces dernières s'appliqueront à tous les mots dérivés ou non. Les règles phonématiques, au contraire, ne s'appliquent pas aux dérivés.

3:12:1. Règles de Structure Accentuelle - Les Quatre Contraintes

Ces règles sont de nature rythmique : elles concernent tout spécialement l'alternance des accents $/ 0 /, / 1 /$, et $/ 2 /$ dans le "squelette" accentuel des mots.

On a établi le tableau (11) des différents types de squelette et de leur population et $1^{\prime}$ on a été amené à poser quatre règles de base dont le degré d'exactitude dans le corpus étudié est de $99,8 \%$ :

- I') Tout squelette comporte un accent /2/

- 2॰) Aucun squelette ne peut comporter trois accents /I/ ou /2/ successifs en position quelconque.:

$-3^{\circ}$ ) Aucun squelette ne peut avoir plus d'un accent /0/ à 1'initiale : donc $* / 00-/$.

$\left.-4^{\circ}\right) L^{\prime} a c c e n t / 1 /$ est toujours suivi d'un accent $/ 0 \%$ Donc :
a) $* /-12-1$
B) $* /-11-/$
c) $* /-1 /$

3.2. 2. Conséquences : L'accent secondaire dans les mots non-composés

Les schémas possibles, c'est-à-dire autorisés par les 4 règles

sont : 


$\begin{array}{ll}\text { I syllabe prétonique } & / 02-/ \\ 2 \text { syllabes prétoniques } & / 102-/ \\ \text { au-delà de } 2 \text { syllabes } & / 0102-/, / 01002-/ \ldots \\ \text { prétoniques } & / 1002-/, / 10002-/ \ldots\end{array}$

Comment est alors régie la position de l'accent / $1 /$ (accent secondaire) au-delà de deux syllabes prétoniques ?

L'étude des mots de ce type triés automatiquement montre clairement que la place de $1^{\prime}$ accent / 1 / est déterminée par l'accentuation propre du formant initial (morphème ou quasi-morphème). On sait que accent $/ 2 /$ et voyelle neutre [ə] sont incompatibles. On a trouvé par ailleurs que jamais la voyelle neutralisée [ə] $n^{\text {te }}$ st attestée dans une syllabe frappée par 1 'accent / $1 /$.

\section{Exemples :}

similar $/ 200 /$

abolish /020/

hypothesis /0200/

[o] accept /02/

[2] academy / $/ 0200 /$ experiment /0200/ apologue /200/ alcohol /200/

microscope $/ 200 /$ homonym $/ 200 /$ heter odyne $/ 2000 /$ epigram $/ 200 /$ similitude /0200/

[x-]abolition /1020/

hypothetical / $10200 /$

[0] acceptance /020/

[æ] academic /1020/ experimental /01020/ a pology /0200/

alcoholic /1020/

microscopy /0200/ homogeneous $/ 1020 /$ heterogeneous / $10020 /$ e pigraphy /0200/ similarity / $10200 /$

[æx-] abolitionist /10200/

hypothetically / $102000 /$

[æ] ] acceptation / $1020 /$

academician /01 020/ experimentation /01 0020/ a pologetically / $0102000 /$ alcoholometer / $100200 /$

microscopic / $1020 /$ homogeneity /100200/ heterogeneity / $100020 /$ epigrammatic/10020/

\subsection{2:13. Les Quasi-Morphèmes}

On vient de voirque le $Q_{0} M_{6}$ conserve un "écho" (accent / $/$ au lieu d'accent $/ 2 /$ ) de son accentuation d'origine quand il est suivi $d^{\text {sune }}$ terminaison forte (et après application des 4 contraintes). En outre, le Q. $\mathrm{M}_{\mathbf{0}}$, conserve son accent principal quand on lui adjoint une terminais on faible.

Exemples: bľbliophile /2000/, phơtograph /200/, cěphalopod/2000/ electrolyse $/ 0200 /$, electrocute $/ 0200 /$, psêudonym $/ 200 /$, atmosphere $/ 200 /$.. (voir aussi les exemples du parag. 3.2.2.). 
Cela signifie naturellement que 〈electro-> par exemple, a une accentuation propre $(/ 020-/)$ et qu'après application des 4 contraintes, et en l'absence d'une terminaison forte, on aura toujours $/ 020 \% /$.

elěctroscope ...

Exemples : elěctrode, elěctrolyse, elěctron, elěctrophone,

En matière d'accentuation on a trouvé que les $\Omega_{.} \mathrm{M}_{\text {. }}$ se pliaient aux mêmes règles que les morphèmes autonomes (11) La règle de "lion" a été évoquée plus haut; une autre règle sera éxroquée dans le paragraphc qui suit. (Parag. 3. 3.12.1):

3.:3.11. Règles phonématiques

Rappelons que ces règles s'appliquent aux formants (morphèmes, quasi-morphèmes et pseudo-morphèmes) et pas aux dérivés. Certaines de ces règles ont été étudiées ailleurs (12) ce qui permettra d’être bref:

3:3:12, Règle des consonnes en contact

L'accentuation est/-20/ quand deux consonnes phonétiques en contact précèdent les terminaisons suivantes :

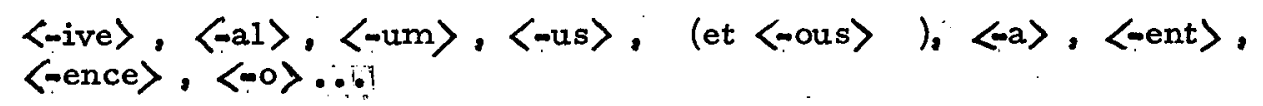

Telle qu'elle est formulée ici, la règle comporte $4 \%$ d'exceptions. On peut réduire ce pourcentage en éliminant $[-\mathrm{kw}-]$ et $[-(\mathrm{s}) \mathrm{tr}]$ de la définition des "clusters". Il ne reste alors que quelques verbes préfixés : misgive, outlive, misdo, undo... et deux ou trois dérivés :

sǔbstance $\rightarrow$ sưbstantive, sìnister $\rightarrow$ sìnistrous

La règle a une portée plus générale qu'il ne parait ici et elle joue également pour les quasi-morphèmes (ex. : hètero-, cěphalon, etc. 6.6$): 1$

Les vérifications sont en cours, mais insistons sur le fait que cette règle ne s'applique pas aux classes homologiques, par exemple <-ingly> (dans răvishingly ... il) ou 〈bly〉 (dans mìserably, disagrěeably ....) ou <-lessness> (dans cărelessness, păssionlessness. i. . ):

4. 1.: Application - Terminaison 〈-ive〉 (résumé)

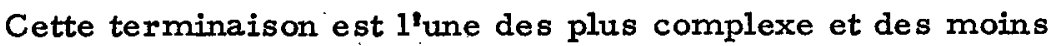
régulières du système accentuel de l'anglais (population 850 mots environ):! 
4:12. Formants

Ce sont les mots de moins de trois syllabes. Ils constituent une faible proportion des mots en 〈-ive〉. On ne rencontre la prononciation [-aiv] que parmi les formants.

1) Monosyllabes

L'opposition phonographématique générale

$\langle$ bit $\rangle[$ bit] $/\langle$ bite $\rangle[$ bait] (cp. $\langle$ not $\rangle /\langle$ note $\rangle$; $\langle$ pal $\rangle \mid\langle$ pale $\rangle ..$ )

donne la relation : $\quad\langle$-ive $\rangle \Rightarrow[$-aiv]

MODELE : dive (five, drive, strive ...)

exceptions : live et give

2) Dissyllabes

VERBES : $/ 02 /$ [-aiv $]$

Modèle : arrive (deprive, connive, contrive, derive ...) exceptions : les préfixés de give et live (forgive, outlive ..66)

ADJECTIFS : $/ 20 / \quad[$-iv $]$

Modèle : native (votive, active, furtive, plaintive, sportive ...) exception : alive /02/ [-aiv]

NOMS :

$\underline{\text { Modèle } 1}$ : archive /20/ [-aiv] (endive (var.1) beehive, Argive ...)

Modèle 2 : olive $/ 20 /[$-iv] (dative, native, motive...)

D'une manière générale l'opposition syntaxique : adjectif /nom n'a pas, en Anglais, de contrepartie phonétique (il en va différemment de 1'opposition verbe/nom). En effet, un mot passe assez facilement d'une classe à $1^{\prime}$ autre (cf. la substantivation det adj. en $\langle-y\rangle,\langle-a b l e\rangle \ldots$... ) La classe $\langle$-ive〉 comporte de nombreux adjectifs mais n'échappe pas à cette règle générale (gerundive, detective, adjective, incentive, infinitive, alternative, genitive, executive, representative ....). Donc, au-delà de deux syllabes, il ne semble pas utile de distinguer les mots suivant leur fonction syntaxique. 


\section{3:- NON-FORMANTS}

Ce sont les mots de plus de deux syllabes. Ils constituent la grande masse des mots en 〈-ive〉. La seule prononciation est [uiv] of Du point de vue de la formation, on peut souvent les rattacher aux mots des classes 〈ory〉et 〈-ion〉.
A/ S-ative
(350 mots)

Trisyllabes :

Classe analogique / $/-200 /$

Modèle : laxat ive (ablative, narrative, locative, relative, talkative, causative ....i).

Exception : creătive /020/ (dérivé de creăte).

Notons que, quand le verbe dérivant est attesté, son accentuation $n^{\prime}$ est pas conservée (nărrative, rělative ...) d d où la décision de décrire cette classe comme analogique ( 1 exception).

Mots de plus de trois syllabes: Classe homologique

Modèle 1 : vindicate $\rightarrow$ vindicative (gěnerative, penetrative ....)

Modèle 2 : consěrve $\rightarrow$ consěrvative (imăginative, quălitative, jǔstificative, intěr pretative ....).

Exceptions : ind Î́cative, altěrnative, interrŏgative, corrělątive, demonstrative, significative (cp. significant).:

Mots sans dérivants: /-200/: prerŏgative, supěrlative, impěrative,

Cas douteux : evŏcative (dérivé de evǒke et non de ěvocate).

B / C $/$ sive $(150$ mots $)$

Classe analogique /.20/

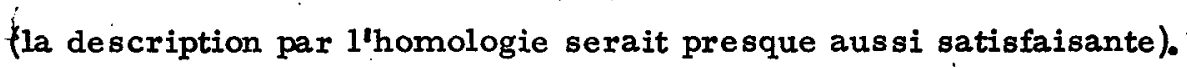

Modèle : exclŭsive (suspěnsive, dissǔasive, progrěssive ....)

Exceptions : pưrposive (dérivé de pưrpose seul formant nominal dans $D$. Jones; Walker donne aussi : récompensive (obsolete ?). 


\section{$C /\left\langle-C_{1} C_{2}\right.$ ive $\rangle(200$ mots $)$}

( 〈-ive〉 précédé de deux consonnes graphiques différentes)

Classe analogique / $/-20 /$

Modèle : instŷnctive (assertive, detective, prescriptive ... )

Exceptions : Z̆djective, sưbstantive (dérivé de sứbstance).

Remarque : une description par homologie poserait le problème de la dérivation dans : incěntive, intellěctive, attěntive ... et celui des ajustements phonématiques peu uniformes dans :

prescribe $\rightarrow$ prescrǔptive; reděem $\rightarrow$ reděmptive presŭme $\rightarrow$ presǔmptive; destrǒy $\rightarrow$ destrǔctive ...

(la diachronie reprend ici ses droits).

$D /$ S-itive〉 et -utive $>-(60$ mots $)$

Classe analogique / $/-200 /$

Modèle : gěnitive (additive, positive, punitive, intuitive, partitive, competitive, infinitive ...).

Exceptions : excľtive [-aitiv] (venant de excǐte), rěsolutive (de rès solute), sưbstitutive (de sǔbstitute) sont des formations homolo. giques exceptionnelles car, d'autre part on a : pretěritive (cp. prěterite), infínitive (cp. Infinite)....

De façon plus générale, cette classe se rattache à la classe phonétique des mots ayant [i] (ou [u] ) en avant-dernière syllabe

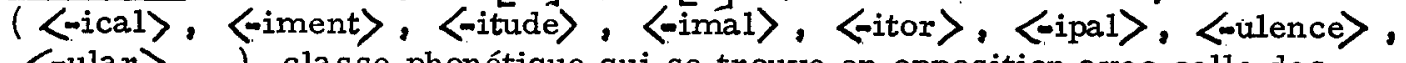
$\langle$-ular $>. .$. ), classe phonétique qui se trouve en opposition avec celle des

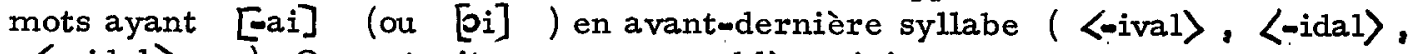
〈-oidal>...). On ne traitera pas ce problème ici.

4. 4. Mots hors-système : khedive [ki'di:v], recitative $\left[\right.$ resit ' $\left.^{\prime} t i: v\right]$.

\section{CONCLUSION}

Tel semble être, dans le cas de $\langle$ ive $\rangle$, le programme, sous forme réduite, des structures maitrisées inconsciemment par l'indigène et;

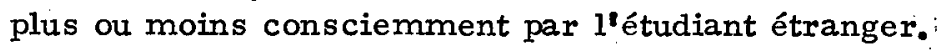


Redisons que d'autres terminaisons sont bien moins complexes.! La terminaison 〈-ive〉 a permis de mettre en relief le conflit si fréquent entre analogie et homologie dans 1'accentuation du mot anglais.

L'analyse automatique qui a permis d'obtenir ces résultats a été subventionnée par le $C_{0} N_{0} \cdot R_{0} S_{0}$ et réalisée à $1^{\imath}$ Institut Blaise Pascal (C. N. R. S. ) Paxis.

\author{
L. GUIERRE \\ Paris, Sorbonne, Mai I 967
}

NOTES

1. 16 mots irréguliers sur 1. 600 (catholic, heretic ....)

2. - On pense à un partage d'influence entre une description néo-bloolfiel dienne et une analyse Chomskiste. En fait, on le verra, il sera nécessaire d'élaborer une synthèse qui pourrait être une réconciliation et dont les parrains pourraient etre A. Martinet, A. A. Hill et I. I.Revzin.

3. - cf. L. Guierre "Eléments ..." op. cit."

4. - voir, ciødessus, parag. I exemple 2, la formule $\left\langle i V^{n}\left(C^{n}\right)\right\rangle$ qui inclut $\langle$-ious $\rangle,\langle-$ ion $\rangle$ etc....

5. - cf. Arnold op. cit:

6. - cf. L. Guierre "Accentuation ..." op. cit.

7.: - Méme si, contrairement à ce que note Jones, il semble préférable d'écrire <phone>/-1/ au lieu de $/-0 /$ et $\langle$ phoner $>/-10 /$ au lieu de $/-00 /$

8. - cf. L. Guierre "Accent secondaire ..." op "i cit.

9. - Le dérivant peut avoir disparu tandis que le dérivé survivait, problème diachronique qui est écarté a priori de la présente étude. 


\section{C $\left\langle-\mathrm{C}_{1} \mathrm{C}_{2}\right.$ ive $\rangle(200$ mots $)$}

( 〈-ive〉 précédé de deux consonnes graphiques différentes)

Classe analogique /-20/

Modèle : instlnctive (assertive, detective, prescriptive ...)

Exceptions : Zujective, sưbstantive (dérivé de sứbstance).

Remarque : une description par homologie poserait le problème de la dérivation dans : incèntive, intellěctive, attěntive ... et celui des ajustements phonématiques peu uniformes dans :

prescr Ǐbe $\rightarrow$ prescrèptive; reděem $\rightarrow$ reděmptive presŭme $\rightarrow$ presúmptive; destrǒy $\rightarrow$ destrǔctive ...

(la diachronie reprend ici ses droits).

D/ 〈-itive〉 et <utive $-(60$ mots $)$

Classe analogique / $/-200 /$

Modèle : gènitive (additive, positive, punitive, intuitive, partitive, competitive, infinitive ....).

Exceptions : excǐtive [-aitiv] (venant de excǐte), rěsolutive (de rěsolute), sưbstitutive (de sǔbstitute) sont des formations homologiques exceptionnelles car, d'autre part on a :

$$
\text { pretěritive (cp. prèterite), infِ̌nitive (cp. Infinite).... }
$$

De façon plus générale, cette classe se rattache à la classe phonétique des mots ayant [i] (ou [u] ) en avant-dernière syllabe $\langle\langle-i c a l\rangle,\langle-i m e n t\rangle,\langle-i t u d e\rangle,\langle-i m a l\rangle,\langle-i t o r\rangle,\langle-i p a 1\rangle,\langle-u l e n c e\rangle$, $\langle-u l a r\rangle . .$. ), classe phonétique qui se trouve en opposition avec celle des mots ayant [-ai] (ou [ 〈-oidal>...). On ne traitera pas ce problème ici.

4. 4. Mots hors-système : khedive [ki'di:v], recitative [,resit a ti:v] .

\section{CONCLUSION}

Tel semble être, dans le cas de $\langle-i v e\rangle$, le programme, sous forme réduite, des structures maitrisées inconsciemment par 1'indigène et,

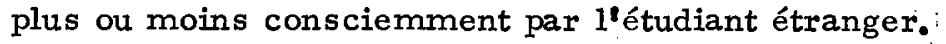


Redisons que d'autres terminaisons sont bien moins complexes. 1 La terminaison 〈-ive〉 a permis de mettre en relief le conflit si fréquent entre analogie et homologie dans l'accentuation du mot anglais.

L'analyse automatique qui a permis d'obtenir ces résultats a été subventionnée par le C.:N.R. So et réalisée à 1'Institut Blaise Pascal (C. N.R. S.) Paris.

\section{GUIERRE}

Paris, Sorbonne, Mai 1967

NOTES

1. 16 mots irréguliers sur 1.600 (catholic, heretic ...)

2. - On pense à un partage d'influence entre une description néo-bloolfiel dienne et une analyse Chomskiste. En faít, on le verra, il sera nécessaire d'élaborer une synthèse qui pourrait être une réconciliation et dont les parrains pourraient être A, iMartinet, A. A. Hill et I, I. Revzin.

3. - cf. L. Guierre "Eléments ..." op. cit,;

4. - voir, ciodessus, parag. I exemple 2, la formule $\left\langle i V^{n}\left(C^{n}\right)\right\rangle$ qui inclut

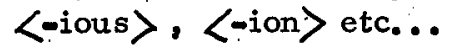

5. - cf. Arnold op. cito:

6.: - cf. L. Guierre "Accentuation ..." op. cit.

7. - Même si, contrairement à ce que note Jones, il semble préférable d'écrire «phone) $/-1 /$ au lieu de $/-0 /$ et $\langle$ phoner $>/-10 /$ au lieu de $/ .00 /$

8. - cf. L. Guierre "Accent secondaire ..." op. cit.

9.: - Le dérivant peut avoir disparu tandis que le dérivé survivait, problème diachronique qui est écarté a priori de la présente étude. 


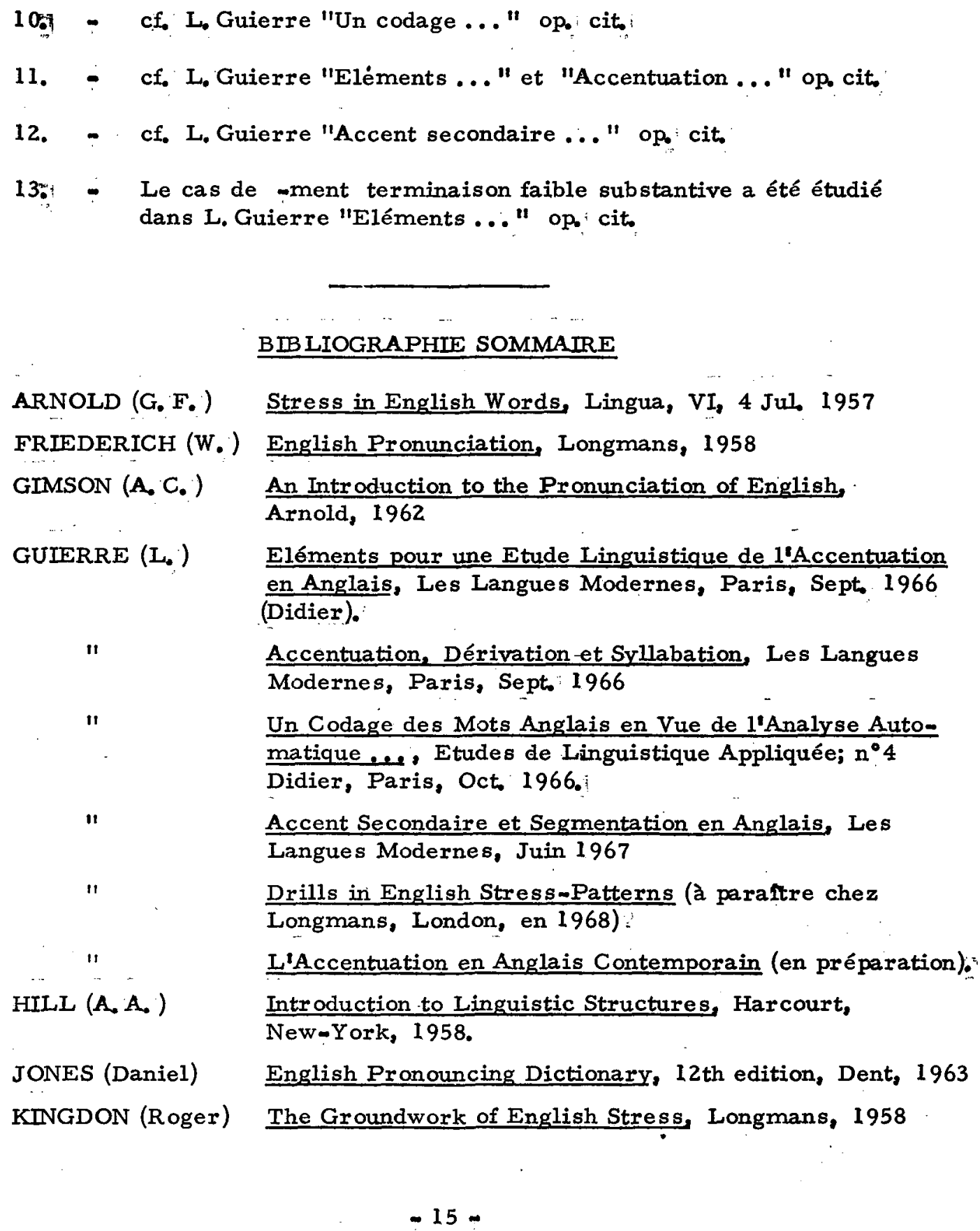




\begin{tabular}{|c|c|}
\hline MAF & The Categories and Types of English Word-Formation, \\
\hline 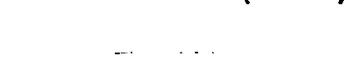 & Otto Harrassowitz, Wiesbaden, 1960 \\
\hline REVZIN (I. I. ) & Models of Language, Methuen, 1966 \\
\hline ROULLIER (U. F.) & $\begin{array}{l}\text { Contribution à 1'Etude Rationnelle de la Prononciation } \\
\text { Anglaise, Orléans, } 1937\end{array}$ \\
\hline WALKER (J.) & The Rhyming Dictionary, Routledge, 1957 \\
\hline WIJK & the Pronunciation of the English Language, \\
\hline
\end{tabular}

